\title{
The neuroprotective effects of pregabalin after cerebral ischemia by occlusion of the middle cerebral artery in rats
}

\author{
JUNEKYUNG LEE ${ }^{1}$, CHANG GU KANG $^{2}$, CHAE RI PARK $^{3}$, IN KYUNG HONG ${ }^{3}$ and DAE YUL KIM ${ }^{2}$ \\ ${ }^{1}$ Department of Rehabilitation Medicine, Hallym University Dongtan Sacred Heart Hospital, \\ Hallym University College of Medicine, Hwaseong, Gyeonggi-do 18450; \\ ${ }^{2}$ Department of Rehabilitation Medicine; ${ }^{3}$ Asan Institute for Life Sciences, Asan Medical Center, \\ University of Ulsan College of Medicine, Seoul 05505, Republic of Korea
}

Received July 22, 2020; Accepted December 3, 2020

DOI: $10.3892 / \mathrm{etm} .2020 .9596$

\begin{abstract}
Activation of presynaptic voltage-gated calcium channels and glutamate release serves a central role in neuronal necrosis after cerebral ischemia. Pregabalin binds to the $\alpha 2-\delta$ subunit of voltage-gated calcium channels and results in reduced glutamate release. The aim of the current study was to evaluate the effect of pregabalin on cerebral outcome following cerebral ischemia using an established rat model. Male Sprague-Dawley rats were randomized to receive oral administration of $5 \mathrm{mg} / \mathrm{kg}$ pregabalin for 1 day (PD1 group) or 5 days (PD5 group), or an equal amount of normal saline for 1 day (SD1 group) or 5 days (SD5 group) after 1 day of middle cerebral artery occlusion (MCAO) and reperfusion. Behavioral tests were assessed at postoperative days 1 and 7. Cerebral infarct volume was measured using a brain MRI scan on days 1 and 7 following surgery. Using immunohistochemistry to detect brain-derived neurotrophic factor (BDNF), histologic examinations of perilesional cortex and ipsilateral hippocampus were performed at postoperative day 7. BDNF-positive immunostaining was more abundant in the perilesional cortex of mice of the PD1 group compared with mice of the SD1 group $(\mathrm{P}=0.001)$. In the ipsilateral
\end{abstract}

Correspondence to: Dr Dae Yul Kim, Department of Rehabilitation Medicine, Asan Medical Center, University of Ulsan College of Medicine, 88, Olympic-ro 43-Gil, Songpa-gu, Seoul 05505, Republic of Korea

E-mail: dykimsmart@gmail.com

Abbreviations: BDNF, brain-derived neurotrophic factor; CCA, common carotid artery; CL, contralateral; CNS, central nervous system; FOV, field of view; GABA, gamma aminobutyric acid; ICA, internal carotid artery; MCA, middle cerebral artery; MCAO, middle cerebral artery occlusion; MRI, magnetic resonance imaging; NMDA, N-methyl-D-aspartate; PBS, phosphate buffered saline; TE, time to echo; TR, time to repeat; VGCC, voltage-gated calcium channels

Key words: brain ischemia, pregabalin, neuroprotection, behavior rating scale, brain-derived neurotrophic factor hippocampus, greater BDNF-positive staining was present in the PD5 group compared with the SD5 group ( $\mathrm{P}=0.04)$. No statistically significant differences were indicated for behavioral tests or cerebral infarct volume between the PD1 and SD1 groups or the PD5 and SD5 groups. In conclusion, treatment with pregabalin beneficially impacts BDNF expression and histologic cerebral outcome in rats after cerebral ischemia.

\section{Introduction}

Cerebral infarction (commonly known as stroke) is a leading cause of disability (1). In recent years, the development of acute medical treatments for stroke has led to a reduction in stroke mortality, but serious disability remains high in many stroke survivors (2). Neurological deficits after a stroke seriously affect quality of life (3).

Neuronal cell death occurs immediately after stroke. Stroke results in massive cell death in the infarct core and severe damage to neurons in the periinfarct area (4). Although periinfarct and penumbral tissues are present in a structurally intact state, they possess dysfunctional metabolism and ion homeostasis (5). Excitatory neurotransmitters released after stroke overexcite tissues around the brain lesion, making the surrounding neurons vulnerable to external stimuli (6). Glutamate is the major excitatory neurotransmitter of the brain and binds to N-methyl-D-aspartate (NMDA) receptors. It is also involved in secondary processes that lead to neuronal death after traumatic insult $(7,8)$. Neuroprotective agents for treatment of acute stroke have received much attention and are expected to effectively protect vulnerable neurons and save ischemic penumbra (9). Several neuroprotective strategies target acute processes, including excitotoxicity, glutamate release, activation of NMDA receptors, calcium ion influx, and apoptosis leading to early cell death in the infarct core and periinfarct area (4).

Gamma aminobutyric acid (GABA) is a major inhibitory neurotransmitter in the central nervous system (CNS) that inhibits depolarization- and ischemia-induced glutamate release (9). GABA receptor agonists are conventional sedatives with neuroprotective effects in decreasing infarct size and improving functional outcomes in animal models of cerebraovascular disease. However, their sedative effects may 
be harmful, so their wider application in patients with acute stroke have been limited (9).

Pregabalin is structurally derived from GABA, and its mechanism of action is associated with binding to the alpha $_{2}$-delta $(\alpha 2-\delta)$ subunit of voltage-gated calcium channels (VGCC). Pregabalin reduces abnormal neuronal excitability by reducing the release of neurotransmitters including glutamate from synapses in neural tissue (10). As a medication, it is used to treat epilepsy and causes of neurogenic pain, such as fibromyalgia, diabetic neuropathy, and complex regional pain syndrome $(10,11)$.

The neuroprotective effects of pregabalin on stroke outcome have been reported in a mouse model (11). Intraperitoneal administration of pregabalin within 30-90 min of stroke reduces cerebral infarction and improves neurological function after $6 \mathrm{~h}$ (11). However, the administration of drugs within 90 min post-stroke is difficult to effectively translate clinically. In addition, their study focused on data collection too early to assess stroke function, making it difficult to determine drug effectiveness on stroke recovery or possibly delayed neurological damage. Therefore, we designed a study to evaluate the effect of pregabalin administered 1 day after cerebral ischemia on cerebral outcome in rats.

The aim of this study was to investigate the effect of pregabalin on cerebral outcome after cerebral ischemia through a middle cerebral artery occlusion (MCAO) rat model by histologic examination, behavioral tests, and magnetic resonance imaging (MRI).

\section{Materials and methods}

Animals. All animal procedures were performed with approval by the Institutional Animal Care and Use Committee in Asan Medical Center (2014-12-212). Male Sprague-Dawley rats weighing 250-280 g and 8 weeks of age were allowed food and water ad libitum and habituated under conditions of a $12 \mathrm{~h}$ light/dark cycle (lights on at 07:00 a.m.) at a temperature of $22^{\circ} \mathrm{C}$ with appropriate humidity. Rats were randomized into one of four groups: Oral administration of $5 \mathrm{mg} / \mathrm{kg}$ pregabalin (Pfizer Pharmaceuticals, New York, NY, USA) for 1 day (PD1, $\mathrm{n}=10$ ), or 5 days (PD5, $\mathrm{n}=10$ ) after MCAO surgery or an equal amount of normal saline administered 1 day $(\mathrm{SD} 1, \mathrm{n}=7)$ or 5 days (SD5, $\mathrm{n}=7$ ) after MCAO surgery.

Occlusion of the middle cerebral artery in rat. To generate a rat model of ischemic stroke, we performed transient occlusion of the right middle cerebral artery (MCA) using the intraluminal filament technique as previously described (12). Inhalation anesthesia was induced in rats with 5\% isoflurane (JW Pharm, Seoul, South Korea) and maintained with 3\% isoflurane. Body temperature was continuously monitored and maintained at $37^{\circ} \mathrm{C} \pm 0.5^{\circ} \mathrm{C}$ using a heating pad during surgery. An incision was made along the ventral midline of the neck, and the right common carotid artery (CCA), external carotid artery, and internal carotid artery (ICA) were exposed. A silicone-coated monofilament nylon suture was inserted into the right CCA through a small incision made in the proximity of the carotid bifurcation, passed through the ICA, and advanced to the right MCA. After 30 min of transient MCAO, reperfusion was achieved by filament withdrawal.
Behavioral tests. Behavioral tests were performed at 1 and 7 days after MCAO/reperfusion surgery. Tests were conducted by a rater who was blind to the treatment. A wire hang test is used to evaluate motor function in rodent models of CNS disorders (13). The test began with the animal hanging from an elevated wire mesh grid $(15 \times 25 \mathrm{~cm})$. The wire mesh grid was then inverted, so that the rat was suspended from the wire mesh. The latency to fall was recorded. Five repetitions were performed with a 3-min interval between repeats and averaged. We also performed the Garcia test, which is a composite neurological test to evaluate various sensorimotor deficits after MCAO in rats $(14,15)$, and includes the following six tests: Spontaneous activity, symmetry in four limb movement, forepaw outstretching, climbing, body proprioception, and response to vibrissae touch. Each test item was scored between 0-3 points and summed to a maximum score of 18 points, with a higher score indicating better function. For the balance beam test $(16,17)$, two square bars $1.5 \mathrm{~cm}$ in width and $40 \mathrm{~cm}$ in height were used as bilateral pillars, and a cylindrical rod $2 \mathrm{~cm}$ in diameter and $60 \mathrm{~cm}$ in length was placed horizontally $40 \mathrm{~cm}$ above the floor, which was covered by a foam pad. Rats were placed in the center of the cylindrical rod, and the time to fall from the rod was measured up to a maximum of $30 \mathrm{sec}$. Testing was repeated four times and averaged for each rat.

Brain MRI. Brain MRI was performed at days 1 and 7 after $\mathrm{MCAO} /$ reperfusion. For MRI, inhalation anesthesia was performed for all rats with 2.0-2.5\% isoflurane in a 1:2 mixture of $\mathrm{O}_{2}: \mathrm{N}_{2} \mathrm{O}$ using a mask. A 9.4T/160 mm animal MRI system (Agilent Technologies, Santa Clara, CA, USA) was used. A $72 \mathrm{~mm}$ birdcage volume coil was used for excitation, and a four-channel phased array surface coil was used as a receiving coil. Rat body temperature was monitored and maintained at $37^{\circ} \mathrm{C} \pm 0.5^{\circ} \mathrm{C}$ using an air heater system.

Infarction of the right hemisphere was confirmed by T2-weighted and diffusion-weighted MRI. T2-weighted imaging was obtained with spin-echo sequence and the following parameters: Field of view $(F O V)=30 \times 30 \mathrm{~mm}$; slice thickness $=1.0 \mathrm{~mm}$; no gap; time to repeat $(\mathrm{TR})=4,000 \mathrm{~ms}$; effective time to echo $(\mathrm{TE})=32.95 \mathrm{~ms}$; k-zero=3; echo spacing=10.98 ms; 32 segments; echo train length=8; average $=1$; and matrix size $=256 \times 256$. Diffusion-weighted imaging was obtained by a four-shot spin-echo-based echo planar imaging sequence with the following parameters: $\mathrm{TR}=3,750 \mathrm{~ms}$; TE=46.22 ms; 96x96 matrix; and an encoding scheme of 30 gradient directions with a b-value of 1,000 $\mathrm{s} / \mathrm{mm}^{2}$. T2-weighted and diffusion-weighted images confirmed right hemisphere infarction. By measuring the volume of infarct-induced right cerebral hemisphere (infarct, or inf) and normal-side cerebral hemisphere (contralateral, or CL) on T2-weighted images, the degree of stroke was calculated by the following formula (Fig. 1) (18): Infarct volume (\%)=volume (inf-volume (CL)/volume (CL) x100.

Immunohistochemistry. Rats were sacrificed 7 days after $\mathrm{MCAO} /$ reperfusion. Rats were anesthetized with 5\% isoflurane and transcardially perfused with $100 \mathrm{ml}$ phosphate buffered saline (PBS) and $100 \mathrm{ml}$ of $4 \%$ paraformaldehyde in $0.1 \mathrm{M}$ PBS. Brains were removed and fixed in $4 \%$ paraformaldehyde. Fixed specimens were embedded in paraffin 
Table I. Infarct volume, behavioral test scores, and histologic data after 1 day administration of pregabalin or normal saline.

\begin{tabular}{|c|c|c|c|}
\hline Test & Normal saline 1 day $(n=7)$ & Pregabalin 1 day $(n=10)$ & P-value \\
\hline Wire hang test (POD 1, sec) & $0.18 \pm 0.37$ & $0.90 \pm 0.94$ & 0.137 \\
\hline Garcia test (POD 1) & $3.86 \pm 1.57$ & $5.30 \pm 2.95$ & 0.244 \\
\hline Beam balance test (POD 1, sec) & $0.75 \pm 1.14$ & $2.55 \pm 3.32$ & 0.153 \\
\hline Infarct volume (POD 1, \%) & $43.2 \pm 9.4$ & $37.5 \pm 25.6$ & 0.810 \\
\hline Number of BDNF-positive cells in perilesional cortex & $85.77 \pm 9.58$ & $114.00 \pm 7.03$ & $0.001^{\mathrm{a}}$ \\
\hline Number of BDNF-positive cells in ipsilateral hippocampus & $127.63 \pm 17.28$ & $137.64 \pm 13.66$ & 0.282 \\
\hline
\end{tabular}

${ }^{a}$ Mann-Whitney test. BDNF, brain-derived neurotrophic factor; POD, postoperative day.
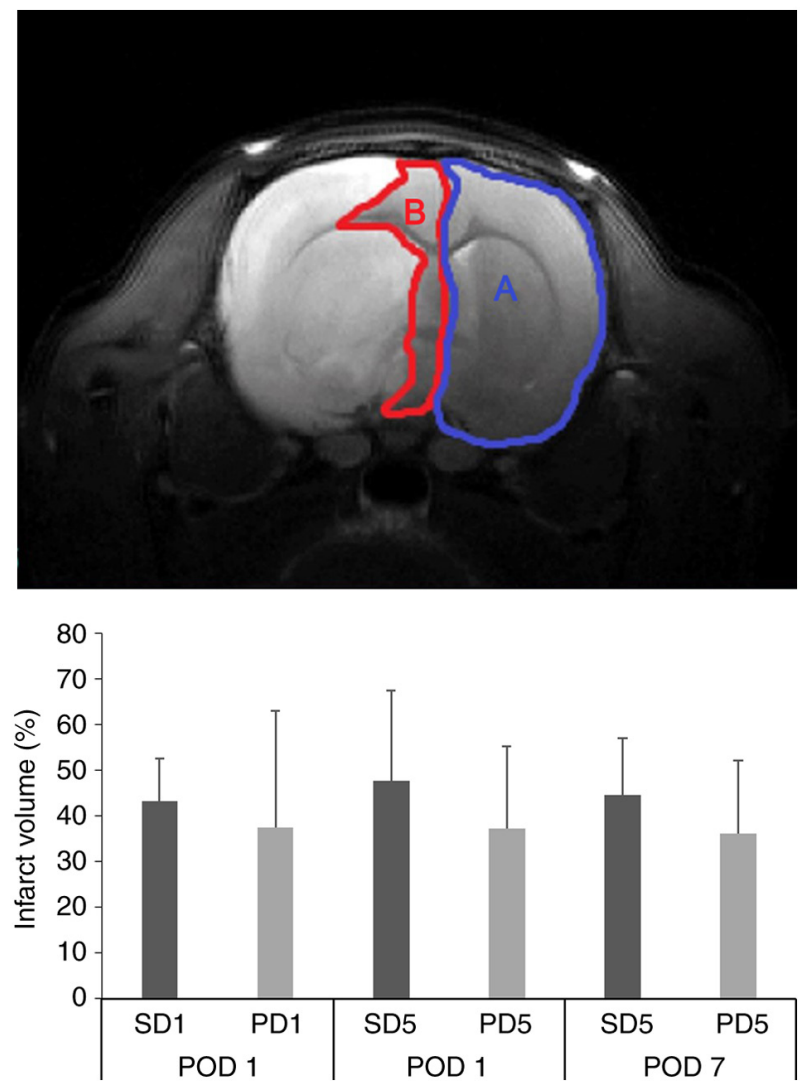

Figure 1. Brain infarct volume showing contralateral and ipsilateral normal areas. Measurements of brain infarct volume and comparisons of brain infarct volumes between pregabalin-administered and control rats with MCAO. Infarct volume $(\%)=(\mathrm{A}-\mathrm{B}) / \mathrm{Ax} 100$. A, contralateral normal area; $\mathrm{B}$, ipsilateral normal area; MCAO, middle cerebral artery occlusion; PD1, pregabalin administration for 1 day; PD5, pregabalin administration for 5 days; POD, postoperative day; SD1, normal saline for 1 day; SD5, normal saline for 5 days.

and were cut into $4 \mu \mathrm{m}$-thick sections. Sections were then deparaffinized in xylene, hydrated in a series of graded alcohol, washed with distilled water, and subjected to microwave in citrate buffer ( $\mathrm{pH}$ 6.0) for $10 \mathrm{~min}$. Endogenous peroxidase activity was inhibited by $3 \%$ hydrogen peroxide for $15 \mathrm{~min}$. Primary antibody against BDNF (1:100, Abcam, Cambridge, MA, USA) was incubated overnight at $4^{\circ} \mathrm{C}$ and detected with a Dako EnVision System HRP/DAB kit (Dako, Glostrup, Denmark). Secondary antibodies including a
1:200 dilution of goat anti-rabbit immunoglobulin (Thermo Fisher Scientific, Waltham, MA, USA) were incubated for $1 \mathrm{~h}$ at room temperature. A 40x objective (DFC290; Leica, Heerbrugg, Germany) with Leica Application Suite (version 3.3.0; Leica) were used to digitize immunostained sections.

To determine the number of BDNF-positive stained cells in the brain, five views of the perilesional cortex and the ipsilateral hippocampus were captured in grayscale. Optical densities were measured in five rectangles of $10,000 \mu \mathrm{m}^{2}$ using MCID analysis (evaluation version 7.0; Imaging Research Inc., ON, Canada), and the values were averaged.

Statistical analysis. Statistical analysis was performed using IBM SPSS Statistics for Windows version 21.0 (IBM Corp., Armonk, NY, USA). The Mann-Whitney U-test was used to compare BDNF expression of the perilesional cortex and ipsilesional hippocampus, infarct volume, and behavioral data between groups. Data are expressed as the mean \pm standard deviation. The cutoff for statistical significance was set at $\mathrm{P}<0.05$.

\section{Results}

Behavioral tests and brain infarct volume. Behavioral test scores of rats from the PD1 group showed better results than those of rats from the SD1 group; however, the differences were not statistically significant. Further, no significant differences were found between the PD5 and SD5 groups (Table I and Table II). No differences in brain infarct volume were found between rats receiving pregabalin and their respective saline controls for 1 or 5 days (PD1 vs. SD1 and PD5 vs. SD5; Fig. 1, Table I, and Table II).

Immunohistochemistry. Using immunohistochemistry (Fig. 2), we found more BDNF-positive cells in the perilesional cortex of rats from the PD1 group compared with that of the SD1 group (Table $\mathrm{I}, \mathrm{P}=0.001$ ). In the ipsilateral hippocampus, we found that the number of BDNF-positive cells in the PD1 group was higher than that of the SD1 group, but the difference was not statistically significant $(\mathrm{P}=0.282)$. We also found more BDNF-positive cells in the ipsilateral hippocampus of rats from the PD5 group compared to the SD5 group (Fig. 2 and Table II, $\mathrm{P}=0.04$ ), whereas in the perilesional cortex, although there were more BDNF-positive cells in the PD5 
Table II. Infarct volume, behavioral test scores, and histologic data after 5 days administration of pregabalin or normal saline.

Test Normal saline 5 days $(n=7) \quad$ Pregabalin 5 days $(n=10) \quad$ P-value

Wire hang test, sec

POD 1

$0.50 \pm 0.85$

$0.65 \pm 0.56$

0.249

POD 7

$1.21 \pm 0.88$

$1.20 \pm 1.70$

0.922

Garcia test, score

POD 1

$4.57 \pm 3.15$

$5.90 \pm 3.18$

0.457

POD 7

$7.29 \pm 4.15$

$8.40 \pm 4.79$

0.921

Beam balance test, sec

POD 1

$1.61 \pm 2.82$

$0.80 \pm 0.98$

0.597

POD 7

$1.25 \pm 1.85$

$1.23 \pm 1.53$

0.961

Infarct volume, \%

POD 1

$47.8 \pm 19.8$

$37.2 \pm 18.1$

1.000

POD 7

$44.7 \pm 12.3$

$36.1 \pm 16.1$

0.610

Number of BDNF-positive cells in perilesional cortex

$99.00 \pm 26.83$

$122.34 \pm 31.24$

0.097

Number of BDNF-positive cells in ipsilateral hippocampus

$116.23 \pm 11.91$

$132.36 \pm 14.56$

$0.040^{\mathrm{a}}$

${ }^{a}$ Mann-Whitney test. BDNF, brain-derived neurotrophic factor; POD, postoperative day.
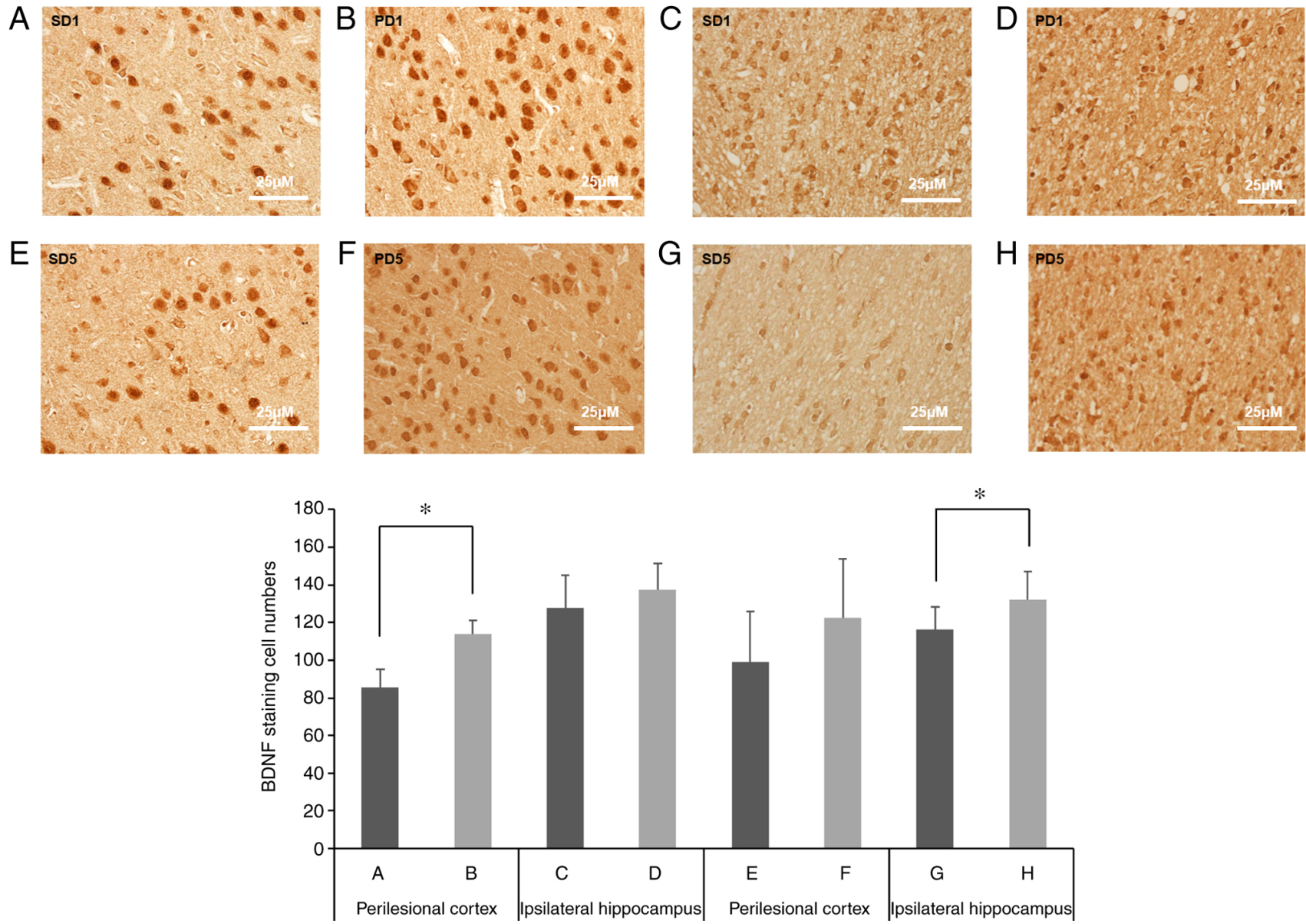

Figure 2. Immunohistochemistry of BDNF in the brain of rats with MCAO. (A) Perilesional cortex section of a rat that received normal saline for 1 day (SD1). (B) Perilesional cortex section of a rat that received pregabalin administration for 1 day (PD1). (C) Ipsilateral hippocampal section of a rat that received normal saline for 1 day (SD1). (D) Ipsilateral hippocampal section of a rat that received pregabalin administration for 1 day (PD1). (E) Perilesional cortex section of a rat that received normal saline for 5 days (SD5). (F) Perilesional cortex section of a rat that received pregabalin administration for 5 days (PD5). (G) Ipsilateral hippocampal section of a rat that received normal saline for 5 days (SD5). (H) Ipsilateral hippocampal section of a rat that received pregabalin administration for 5 days (PD5). "P<0.05. BDNF, brain-derived neurotrophic factor; MCAO, middle cerebral artery occlusion; PD1, pregabalin administration for 1 day; PD5, pregabalin administration for 5 days; POD, postoperative day; SD1, normal saline for 1 day; SD5, normal saline for 5 days. 
group compared with the SD5 group, the difference was not significant $(\mathrm{P}=0.097)$.

\section{Discussion}

In the current study, we evaluated the neuroprotective effect of pregabalin administration 1 and 5 days after MCAO in a rat model of cerebral infarction. Our results show that pregabalin induces a neuroprotective effect by increasing BDNF expression in our MCAO rat model.

Excessive glutamate release from presynaptic nerve endings occurs during and after cerebral ischemia, resulting in persistent calcium influx in cells through postsynaptic NMDA receptors and VGCC. High calcium levels induce neuronal death in the penumbra of ischemic brain (11). Pregabalin specifically binds to the $\alpha 2-\delta$ subunit of VGCC, and its strong binding affinity to this site reduces calcium influx in presynaptic nerve endings, which subsequently leads to the reduced release of excitatory neurotransmitters, such as glutamate and noradrenalin $(11,19)$. It also protects neurons from calcium ion overload during ischemic stroke.

Several previous studies investigated the neuroprotective effects of pregabalin $(11,19-22)$. In a rat model of ischemic stroke, pregabalin administration 30-90 min after transient MCAO and reperfusion reduces infarct volume, neurological deficits, and neuronal damage by $24 \mathrm{~h}$ after stroke (11). The neuroprotective action of pregabalin also contributes to a reduction of calcium/calpain-mediated proteolysis (11). Further, the beneficial effect of pregabalin on functional and histologic cerebral outcome was demonstrated in rats after cardiopulmonary bypass with deep hypothermic circulatory arrest (19). In addition, pregabalin protects against oxidative stress damage after cerebral ischemia and reperfusion by reducing lipid peroxidation and activity of antioxidant enzymes (21). When administered upon reperfusion, pregabalin reduces neuronal death and improves neurological function in a rat model of hyperglycemic stroke (22). Thus, our data support growing evidence of the neuroprotective effect and potential application of pregabalin after cerebral ischemia.

BDNF is a potent modulator beneficial to neuronal function (8). BDNF plays a crucial role in synaptic plasticity in the CNS, stimulating neurons to survive and promoting the growth and differentiation of new neurons (23). The neuroprotective effect of BDNF in stroke models is achieved by anti-apoptotic, anti-inflammatory, and anti-neurotoxic effects, and promotion of neural regeneration (8). In the present study, BDNF was upregulated in the perilesional cortex after administration of pregabalin for 1 day and in the ipsilateral hippocampus after pregabalin administration for 5 days compared with administration of normal saline, suggesting that pregabalin has a neuroprotective effect in rat models of cerebral ischemia. Furthermore, pregabalin may present new possibilities in the promotion of neurotrophic factors in ischemic brain. In addition, our finding that BDNF upregulation was significantly observed in perilesional cortex after administration of pregabalin for 1 day and in the ipsilateral hippocampus after pregabalin administration for 5 days may reflect differences in pregabalin response in motor- and cognition-related brain regions and duration of pregabalin administration. However, further research is needed to investigate this interpretation.
We found no significant difference in cerebral infarct volume between pregabalin and control groups. For behavioral tests, although not statistically significant, the overall score was higher in the pregabalin-administered group than in the control group, with a few exceptions. This results are inconsistent with previous studies that found infarct volume and neurological deficits were reduced $24 \mathrm{~h}$ post-stroke when $5-10 \mathrm{mg} / \mathrm{kg}$ pregabalin was given intraperitoneally 30-90 min after MCAO and reperfusion in rats (11). Similarly, a different study found that intraperitoneal injection of $30 \mathrm{mg} / \mathrm{kg}$ pregabalin $1 \mathrm{~h}$ after MCAO and reperfusion reduced infarct size and improved neurological function as determined by neurobehavioral assessment $24 \mathrm{~h}$ post-stroke in rats with hyperglycemic stroke (22). It is speculated that the dose of pregabalin, route of administration, or time point of outcome measurement may have an effect on these differing results in cerebral infarct volume and neurological function between studies; however, further research is needed to determine whether this is the case.

There are several limitations to this study. First, we did not directly reveal the effect of pregabalin on each specific pathway of neuronal cell death. Although we examined the effects of pregabalin on BDNF expression in the brain of ischemic rats from pathological and functional perspectives, we did not assess the possible underlying molecular mechanism such as the signaling pathway by which pregabalin increases BDNF expression in the brain after ischemia. Second, immunohistochemical staining was not further analyzed semi-quantitatively. Further research to determine BDNF protein levels in the brain by western blotting is needed to identify the mechanistic pathways directly involved in cerebral outcomes as a result of the neuroprotective effects of pregabalin. Third, we did not directly compare the effects of pregabalin administration duration between 1 and 5 days; we only compared the pregabalin-administered group to their respective normal saline control group. However, previous studies have evaluated the effects of single doses of pregabalin; therefore, our study is still informative because we varied the duration of pregabalin administration. Nevertheless, further research is needed to investigate whether the duration of pregabalin administration affects other responses. Finally, the hippocampus is involved in cognitive function, but the behavioral tests performed in this study did not include any relevant cognitive tests.

In conclusion, pregabalin treatment confers a beneficial effect on histologic cerebral outcome in a rat model of cerebral ischemia, and that this effect may be mediated by increased BDNF expression. Our results support the neuroprotective role of pregabalin and its potential use for ischemic cerebrovascular disease. However, because pregabalin did not affect infarct volume or performance on behavioral tests in our study, additional research is warranted.

\section{Acknowledgements}

Not applicable.

\section{Funding}

This study was supported by a grant (2018-454) from the Asan Institute for Life Sciences, Asan Medical Center, Seoul, Korea. 


\section{Availability of data and materials}

The datasets used and/or analyzed in the current study are available from the corresponding author on reasonable request.

\section{Authors' contributions}

JL analyzed the data and wrote the manuscript. CGK designed the study and analyzed the data. CRP and IKH performed the experiments. DYK designed the study and revised the manuscript. JK and DKY confirm the authenticity of all the raw data. All authors read and approved the final manuscript.

\section{Ethics approval and consent to participate}

This study was approved by the Institutional Animal Care and Use Committee in Asan Medical Center in South Korea (2014-12-212) and conducted in strict accordance with the recommendations of the United States National Institutes of Health Guide for the Care and Use of Laboratory Animals (Publication No. 85-23, revised 1996).

\section{Patient consent for publication}

Not applicable.

\section{Competing interests}

The authors declare that they have no competing interests.

\section{References}

1. Duncan PW: Stroke disability. Phys Ther 74: 399-407, 1994.

2. Brewer L, Horgan F, Hickey A and Williams D: Stroke rehabilitation: Recent advances and future therapies. QJM 106: 11-25, 2013.

3. Sturm JW, Donnan GA, Dewey HM, Macdonell RA, Gilligan AK, Srikanth V and Thrift AG: Quality of life after stroke: The north East melbourne stroke incidence study (NEMESIS). Stroke 35: 2340-2345, 2004.

4. Kumar A and Kitago T: Pharmacological enhancement of stroke recovery. Curr Neurol Neurosci Rep 19: 43, 2019.

5. Sist B, Fouad K and Winship IR: Plasticity beyond peri-infarct cortex: Spinal up regulation of structural plasticity, neurotrophins, and inflammatory cytokines during recovery from cortical stroke. Exp Neurol 252: 47-56, 2014.

6. Risedal A, Zeng JS and Johansson BB: Early training may exacerbate brain damage after focal brain ischemia in the rat. J Cereb Blood Flow Metab 19: 997-1003, 1999.

7. Humm JL, Kozlowski DA, Bland ST, James DC and Schallert T: Use-dependent exaggeration of brain injury: Is glutamate involved? Exp Neurol 157: 349-358, 1999.
8. Chen A, Xiong LJ, Tong Y and Mao M: The neuroprotective roles of BDNF in hypoxic ischemic brain injury. Biomed Rep 1: 167-176, 2013.

9. Liu J, Zhang J and Wang LN: Gamma aminobutyric acid (GABA) receptor agonists for acute stroke. Cochrane Database Syst Rev 10: CD009622, 2018.

10. Taylor CP, Angelotti T and Fauman E: Pharmacology and mechanism of action of pregabalin: The calcium channel alpha $a_{2}$-delta (alpha ${ }_{2}$-delta) subunit as a target for antiepileptic drug discovery. Epilepsy Res 73: 137-150, 2007.

11. Yoon JS, Lee JH, Son TG, Mughal MR, Greig NH and Mattson MP: Pregabalin suppresses calcium-mediated proteolysis and improves stroke outcome. Neurobiol Dis 41: 624-629, 2011.

12. Sasaki M, Honmou O and Kocsis JD: A rat middle cerebral artery occlusion model and intravenous cellular delivery. Methods Mol Biol 549: 187-195, 2009.

13. Olivan S, Calvo AC, Rando A, Munoz MJ,Zaragoza P and Osta R Comparative study of behavioural tests in the SOD1G93A mouse model of amyotrophic lateral sclerosis. Exp Anim 64: 147-153, 2015.

14. Garcia JH, Wagner S, Liu KF and Hu XJ: Neurological deficit and extent of neuronal necrosis attributable to middle cerebral artery occlusion in rats. Statistical validation. Stroke 26: 627-634; discussion 635, 1995.

15. Hartman R, Lekic T, Rojas H, Tang J and Zhang JH: Assessing functional outcomes following intracerebral hemorrhage in rats. Brain Res 1280: 148-157, 2009.

16. Combs DJ and D'Alecy LG: Motor performance in rats exposed to severe forebrain ischemia: Effect of fasting and 1,3-butanediol. Stroke 18: 503-511, 1987.

17. DeGraba TJ, Ostrow P, Hanson S and Grotta JC: Motor performance, histologic damage, and calcium influx in rats treated with NBQX after focal ischemia. J Cereb Blood Flow Metab 14: 262-268, 1994.

18. Frey LC,Hellier J, Unkart C, Lepkin A, Howard A, Hasebroock K, Serkova N, Liang L, Patel M, Soltesz I and Staley K: A novel apparatus for lateral fluid percussion injury in the rat. J Neurosci Methods 177: 267-272, 2009.

19. Shim JK, Ma Q, Zhang Z, Podgoreanu MV and Mackensen GB: Effect of pregabalin on cerebral outcome after cardiopulmonary bypass with deep hypothermic circulatory arrest in rats. J Thorac Cardiovasc Surg 148: 298-303, 2014.

20. Ha KY, Kim YH, Rhyu KW and Kwon SE: Pregabalin as a neuroprotector after spinal cord injury in rats. Eur Spine J 17: 864-872, 2008

21. Asci S, Demirci S, Asci H, Doguc DK and Onaran I: Neuroprotective effects of pregabalin on cerebral ischemia and reperfusion. Balkan Med J 33: 221-227, 2016.

22. Song Y, Jun JH, Shin EJ, Kwak YL, Shin JS and Shim JK: Effect of pregabalin administration upon reperfusion in a rat model of hyperglycemic stroke: Mechanistic insights associated with high-mobility group box 1. PLoS One 12: E0171147, 2017.

23. Hu Y, Guo TC, Zhang XY, Tian J and Lu YS: Paired associative stimulation improves synaptic plasticity and functional outcomes after cerebral ischemia. Neural Regen Res 14: 1968-1976, 2019.

This work is licensed under a Creative Commons Attribution-NonCommercial-NoDerivatives 4.0 International (CC BY-NC-ND 4.0) License. 